6

\title{
Therapeutic Potential of Dronabinol: A Systematic Review of Human Trials
}

\author{
Julia Lewandowska1 ${ }^{1}$, Jan Maciej Kapała ${ }^{1}$, Łukasz Puchała ${ }^{2}$ \\ ${ }^{1}$ Student, Uniwersytet Warmińsko-Mazurski, Olsztyn, Polska \\ ${ }^{2}$ Katedra Farmakologii i Toksykologii, Uniwersytet Warmińsko-Mazurski, Olsztyn, Polska
}

Farmacja Polska, ISSN 0014-8261 (print); ISSN 2544-8552 (on-line)

Adres do korespondencji

Łukasz Puchała, Katedra Farmakologii

i Toksykologii, Uniwersytet Warmińsko-Mazurski, ul. Warszawska 30, 10-082 Olsztyn, Polska; e-mail: lukasz.puchala@uwm.edu.pl

\section{Źródla finansowania}

Nie wskazano źródeł finansowania.

Konflikt interesów

Nie istnieje konflikt interesów.

Otrzymano: 2021.08.24

Zaakceptowano: 2021.09.29

Opublikowano on-line: 2021.09.30

DOI

$10.32383 /$ farmpol/142693

ORCID

Julia Lewandowska (ORCID id: 0000-0002-3493-2047) Jan Maciej Kapała (ORCID id: 0000-0003-4019-851X)

Łukasz Puchała (ORCID id: 0000-0003-0009-947X)

\section{Copyright}

(c) Polskie Towarzystwo Farmaceutyczne

To jest artykuł o otwartym dostępie,

na licencji CC BY NC (c) (1) ()

https://creativecommons.org/licenses/by-nc/4.0/
Therapeutic Potential of Dronabinol: A Systematic Review of Human Trials

Dronabinol is an isomer of tetrahydrocannabinol that seems to have the potential for both somatic and psychiatric disorders treatment. The purpose of this review is to provide an overview of collected articles with emphasis on the efficacy and safety of dronabinol pharmacotherapy. A literature review was performed in May 2021. Three databases (PubMed, ClinicalTrials, and Cochrane Library) were searched using the following terms: "Dronabinol", "Marinol", "Syndros" and "Namisol" for published studies in English in 2016-2021. Finally, 29 articles were included: 27 randomized controlled clinical trials (RCTs), one open-label clinical trial, and one combination of both previously mentioned designs. According to reviewed studies, dronabinol could be a therapeutic option for cannabis use disorder, Obstructive Sleep Apnea (OSA), Tourette Syndrome (TS), relieving stress, and anorexia treatment.

Keywords: Dronabinol, Systematic review, Clinical trials, Clinical pharmacy.

() Farm Pol, 2021, 77(8): 477-490 


\section{Introduction}

Dronabinol ((6aR,10aR)-6,6,9-trimethyl-3-pentyl-6a,7,8,10a tetrahydrobenzo[c]chromen-1-ol), a specific delta-9-tetrahydrocannabinol (THC) isomer ((6aR-cis)-Isomer) is the main and most active substance found in Cannabis sativa. Because of its administration form, it is also called oral-THC. According to its artificial chemical production, it belongs to a group of substances called synthetic cannabinoids (like e.g. nabilone) which with both phytocannabinoids and endocannabinoids constitute the term medical marijuana [1].

Cannabinoids belong to a class of biological compounds that affect the endocannabinoid system (ECS). This system is a complex network, which plays a role in hemostasis including gastrointestinal, reproductive, immune, neuropsychiatric, cardiovascular, and pulmonary system activity. Therefore, it plays an enormous role in e.g. pain perception, memory, movement, appetite, metabolism, lacrimation, and salivation function [2]. Cannabinoids affect human organisms by stimulating two receptors - cannabinoid receptor type 1 (CB1R) and type 2 (CB2R) [3]. CB1R is abundant mostly in the central nervous system (CNS), especially in the basal ganglia, cerebellum, hippocampus, and cortex, but also present in the cardiovascular system, reproductive systems, and gastrointestinal tract. CB2Rs, by contrast, mostly occur in the peripheral nervous system and immunocompetent tissues [4]. CB2Rs are mostly coupled to $G_{i / o}$ proteins, through which they inhibit the activity of adenylate cyclases and stimulate mitogen-activated protein kinases (MAPK). Moreover, CB1Rs stimulation inhibits voltage-activated calcium channels and activates inwardly rectifying potassium channels [5]. Dronabinol has a similar affinity to CB1 and CB2 receptors. However, it demonstrates its effects mostly via partial agonism at the CB1R, so it produces affective, sensory, somatic, and cognitive effects in the CNS and has central sympathomimetic activity [6].

Dronabinol capsules are mostly absorbed after a single oral dose and reach an onset of action after approximately 0.5-1 hours and peak effect from 2 to 4 hours. Up to $1 / 5$ of the administered dose obtain the systemic circulation in view of the combined effects of first-pass hepatic metabolism and high lipid solubility. Dronabinol is metabolized mainly in the liver by several reactions catalyzed by Cytochrome P450 enzymes. 11-hydroxy-delta-9-tetrahydrocannabinol (11-OH-THC) is the primary active metabolite, which produces the somatic and psychic effect, while 11-nor-9-carboxy-delta-9-tetrahydrocannabinol (THC- $\mathrm{COOH}$ ) is the final inactive one. The last-mentioned biotransformation product is excreted in both feces and urine. Because of dronabinol's metabolism, urine levels may be used as a marker to determine the bioavailability of this substance. However, THC in marijuana has the same final product of metabolism - THC-COOH so it is impossible to measure the effects of cannabinoid use disorder treatment through urine samples tests. Prolonged high-dose administration may cause addiction, although its uncommon and mostly physiological and psychological dependence should be considered as an alarming characteristic [1].

Dronabinol received initial US Food and Drug Administration approval in 1985 as an oral capsule formulation, but as late as 2016 was approved as an oral solution [7]. At present, it is the only prescription drug available under the brand names of "Marinol" (capsules; $2.5 \mathrm{mg}, 5 \mathrm{mg}, 10 \mathrm{mg}$ THC), "Syndros" (solution; $5 \mathrm{mg}$ THC), and "Namisol" (capsules) in several countries including Austria, France, Spain, Sweden, Switzerland, USA, Germany, Australia [1, 8-10]. Actual drug indications of dronabinol include treatment of AIDS-related wasting, anorexia, chemotherapy-induced nausea, and vomiting in patients who have failed to respond adequately to conventional antiemetic treatments and post-traumatic stress disorder (PTSD) $[8,11]$.

This systematic review was performed in order to highlight both the positive and negative impact of dronabinol pharmacotherapy on human organism functions. It also reports the current evidence, literature, and experiences of therapy scheme and dosage strategy of dronabinol

Table 1. Dronabinol - summary and structural formula [1].

$\begin{array}{lll}\text { Name } & \text { Other names } \\ \text { Dronabinol } & \text { delta9-tetrahydrocannabinol, delta9-THC, Marinol, Syndros, Namisol }\end{array}$

Structural formula made in "MedChem Designer (TM) version 5.5.0.11 64-bit edition". 
in specific disease entities as well as efficacy and safety reports of dronabinol intervention.

\section{Materials and methods}

\section{Study eligibility criteria}

The literature review and assessment were performed systematically by two authors in May 2021. Three databases (PubMed, ClinicalTrials, and Cochrane Library) were searched using the following terms: "Dronabinol", "Marinol", "Syndros" and "Namisol" for published studies in English. We included human studies published in 2016-2020 with no demographic limitation. The authors did not exclude trials due to the number, sex, age, race, or country origin of participants. Drug intervention of incorporated studies concerned both, the monotherapy of dronabinol and combination therapies. Chosen research must have reported dosage and dosage schemes to be included. Only full-text studies were accessed for review, although with no limitation due to study design. Eventual disagreements were solved through consensus.

\section{Data collection}

The data extraction was performed independently by two reviewers with emphasis on name or author of the study, year of publication, study population and its characteristic (conditions and symptoms), study design, drug intervention (product/brand), dosage and therapy duration, primary outcome, outcome measures, effects of treatment, adverse effects (AEs), side effects and toxicity. Possible incompatibilities were elucidated through consensus.

\section{Synthesis}

According to the high heterogeneity of the included studies, in the discussion, there was no synthesis in the form of a meta-analysis performed and the results of each study were performed through narrative synthesis. Clinical heterogeneity was assessed by grouping studies by indication of dronabinol pharmacotherapy and disease entity. The discussion also mentions ongoing clinical trials that allow for a more complete analysis of the therapeutic potential of dronabinol.

In order to evaluate the certainty of the evidence, the Grading of Recommendations Assessment,

Figure 1. PRISMA flow diagram of literature search and selection process.

PRISMA: Preferred Reporting Items for Systematic Reviews and Meta-Analyses. Diagram prepared in "MeeSoft Diagram Designer version 1.21".
Development, and Evaluation (GRADE) was used across all the dominants: methodological limitations of the studies, indirectness, imprecision, inconsistency, and publication bias [12].

\section{Results}

\section{Study selection and characteristics}

In this review, we identified 98 studies of which 82 were assessed for full-text eligibility (Figure 1). Finally, 27 randomized controlled clinical trials (RCTs), one open-label clinical trial, and one combination of both previously mentioned methods. Among 28 RCTs, 16 were parallel RCTs and 12 crossover RCTs. The administration of dronabinol varied from single doses to chronic administration, up to 32 weeks. The monotherapy of dronabinol was used in the majority of the studies except for two studies using a combination of dronabinol and lofexidine or dronabinol and clonidine.

Out of the selected articles, six were related to the treatment of cannabis use disorder, one focused on the opioid addiction treatment, nine was about analgesia, five concerned the impact on brain function, one analyzed the sleep improvement in Obstructive Sleep Apnea (OSA), one examined relieving nausea and

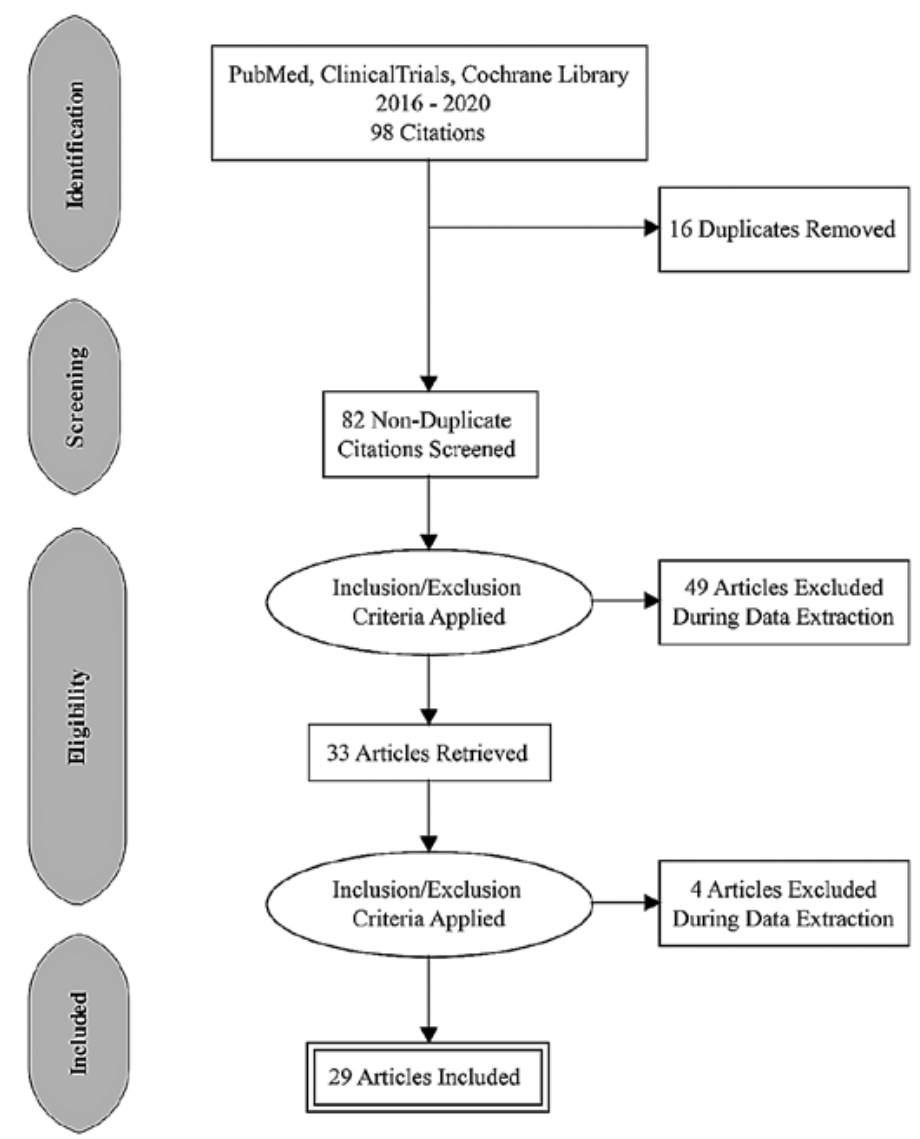


vomiting, one concerned alleviating symptoms in Tourette Syndrome (TS), one concerned relieving stress, one was related to an increase food intake in anorexia, one examined the relieving dementia neuropsychiatric symptoms, one was about an abuse potential and one concerned about the impact on hemodynamics. Results of the review are presented in Table 2 providing a summary of the included studies and their outcomes.

Table 2. Summary of included studies: Therapeutic potential of dronabinol.

\begin{tabular}{|c|c|c|c|c|c|c|c|}
\hline $\begin{array}{l}\text { Name/author } \\
\text { and year of } \\
\text { publication }\end{array}$ & Population & Study design & $\begin{array}{l}\text { Drug intervention } \\
\text { and scheme }\end{array}$ & Primary outcome & Measures & Effects & $\begin{array}{l}\text { Adverse effects, } \\
\text { side effects, and } \\
\text { toxicity }\end{array}$ \\
\hline \multicolumn{8}{|c|}{ Cannabis use disorder } \\
\hline $\begin{array}{l}\text { Levin et al. } \\
2016[13]\end{array}$ & $\begin{array}{l}\text { Cannabis } \\
\text { smokers }(n= \\
122)\end{array}$ & $\begin{array}{l}\text { RCT parallel } \\
\text { double-blind }\end{array}$ & $\begin{array}{l}\text { Dronabinol } 60 \mathrm{mg} \\
\text { and Lofexidine } \\
1.8 \mathrm{mg} / \mathrm{day} ; \\
10 \text { weeks }\end{array}$ & $\begin{array}{l}\text { Changes in the } \\
\text { number of cannabis } \\
\text { cigarettes smoked } \\
\text { and craving severity }\end{array}$ & TLFB, WDS, MCQ & None & $\begin{array}{l}\text { No serious AEs: } \\
\text { dry mouth }\end{array}$ \\
\hline $\begin{array}{l}\text { NCT01394185 } \\
2017[14]\end{array}$ & $\begin{array}{l}\text { Cannabis } \\
\text { smokers }(n=13)\end{array}$ & $\begin{array}{l}\text { RCT parallel } \\
\text { double-blind }\end{array}$ & $\begin{array}{l}\text { Dronabinol: } \\
120 \mathrm{mg} \text { or } \\
240 \mathrm{mg} / \text { day; } \\
12 \text { days }\end{array}$ & $\begin{array}{l}\text { Changes in number } \\
\text { of cannabis } \\
\text { cigarettes smoked }\end{array}$ & $\begin{array}{l}\text { Marijuana Self- } \\
\text { administration: } \\
\text { Progressive Ratio and } \\
\text { Drug Vs Money Choice }\end{array}$ & $\begin{array}{l}\text { Dronabinol reduced the } \\
\text { number of cannabis cigarettes } \\
\text { smoked during therapy }\end{array}$ & None \\
\hline $\begin{array}{l}\text { NCT00893074 } \\
2017[15]\end{array}$ & $\begin{array}{l}\text { Cannabis } \\
\text { smokers }(n=13)\end{array}$ & $\begin{array}{l}\text { RCT crossover } \\
\text { double-blind }\end{array}$ & $\begin{array}{l}\text { Dronabinol } \\
30 \mathrm{mg} \text { or } 60 \mathrm{mg} \\
\text { or } 120 \mathrm{mg} / \mathrm{day} \text {; } \\
12 \text { days }\end{array}$ & $\begin{array}{l}\text { Effect of marijuana } \\
\text { withdrawal }\end{array}$ & $\begin{array}{l}\text { MWC, VAS subjective } \\
\text { drug effect, HR }\end{array}$ & $\begin{array}{l}\text { Dronabinol reduced cannabis } \\
\text { self-administration and } \\
\text { suppressed withdrawal } \\
\text { symptoms }\end{array}$ & $\begin{array}{l}\text { No serious AEs: } \\
\text { dry mouth }\end{array}$ \\
\hline $\begin{array}{l}\text { NCT01598896 } \\
2018[17]\end{array}$ & $\begin{array}{l}\text { Cannabis } \\
\text { smokers with } \\
\text { schizophrenia } \\
(\mathrm{n}=5)\end{array}$ & $\begin{array}{l}\text { RCT parallel } \\
\text { quadruple- } \\
\text { blind }\end{array}$ & $\begin{array}{l}\text { Dronabinol: } \\
15 \text { mg and } \\
\text { Clonidine } \\
0.2 \text { mg/day; } \\
10 \text { weeks }\end{array}$ & $\begin{array}{l}\text { Changes in the } \\
\text { number of cannabis } \\
\text { cigarettes smoked }\end{array}$ & $\begin{array}{l}\text { Subject self-report } \\
\text { hits of marijuana per } \\
\text { day, MCQ }\end{array}$ & $\begin{array}{l}\text { The combination of dronabinol } \\
\text { and clonidine reduced the } \\
\text { number of cannabis cigarettes } \\
\text { smoked during therapy }\end{array}$ & None \\
\hline $\begin{array}{l}\text { NCT00480441 } \\
2019[18]\end{array}$ & $\begin{array}{l}\text { Cannabis } \\
\text { smokers }(n=12)\end{array}$ & $\begin{array}{l}\text { RCT parallel } \\
\text { double-blind }\end{array}$ & $\begin{array}{l}\text { Dronabinol max } \\
40 \mathrm{mg} / \mathrm{day} \\
3 \text { weeks }\end{array}$ & $\begin{array}{l}\text { Physiological } \\
\text { changes in } \\
\text { response to cue- } \\
\text { induced craving } \\
\text { and tolerability of } \\
\text { treatment }\end{array}$ & fMRI & $\begin{array}{l}\text { Dronabinol reduced the } \\
\text { severity of marijuana craving } \\
\text { during therapy }\end{array}$ & $\begin{array}{l}\text { No serious AEs: } \\
\text { drowsiness, } \\
\text { increased craving }\end{array}$ \\
\hline $\begin{array}{l}\text { Schlienz et al. } \\
2019[20]\end{array}$ & $\begin{array}{l}\text { Cannabis users } \\
(\mathrm{n}=13)\end{array}$ & $\begin{array}{l}\text { RCT crossover } \\
\text { double-blind }\end{array}$ & $\begin{array}{l}\text { Dronabinol } 120 \text { or } \\
180-240 \mathrm{mg} / \\
\text { day; } 12 \text { days }\end{array}$ & $\begin{array}{l}\text { Reduction of } \\
\text { cannabis self- } \\
\text { administration }\end{array}$ & $\begin{array}{l}\text { Cannabis } \\
\text { consumption, } \\
\text { cannabis vs money } \\
\text { choice condition, } \\
\text { MWC, VAS sleep, } \\
\text { HR, BP }\end{array}$ & $\begin{array}{l}\text { Dronabinol reduced cannabis } \\
\text { consumption, craving, and } \\
\text { improved quality of life }\end{array}$ & None \\
\hline \multicolumn{8}{|c|}{ Opioid addiction } \\
\hline $\begin{array}{l}\text { Lofwall et al. } \\
2016[23]\end{array}$ & $\begin{array}{l}\text { Opioid } \\
\text { dependent } \\
\text { patients }(n=12)\end{array}$ & $\begin{array}{l}\text { RCT parallel } \\
\text { double-blind }\end{array}$ & $\begin{array}{l}\text { Dronabinol } \\
5 \text { or } 10 \text { or } \\
20 \text { or } 30 \mathrm{mg} \text { or } \\
\text { Oxycodone } 30 \text { or } \\
60 \mathrm{mg} / \mathrm{session} ; \\
7 \text { sessions }\end{array}$ & $\begin{array}{l}\text { Changes in } \\
\text { suppression of } \\
\text { opioid withdrawal } \\
\text { symptoms }\end{array}$ & $\begin{array}{l}\text { Opioid adjective } \\
\text { scale, VAS, SOWS, } \\
\text { CPT, OOWS, time } \\
\text { estimation and } \\
\text { street value task, } \\
\text { physiological } \\
\text { measures }\end{array}$ & $\begin{array}{l}\text { Dronabinol presented little } \\
\text { withdrawal effects of limited } \\
\text { duration }\end{array}$ & $\begin{array}{l}\text { Nervousness and } \\
\text { tachycardia }\end{array}$ \\
\hline \multicolumn{8}{|l|}{ Pain } \\
\hline $\begin{array}{l}\text { NCT00743119 } \\
2017[26]\end{array}$ & $\begin{array}{l}\text { Cannabis } \\
\text { smokers }(n=30)\end{array}$ & $\begin{array}{l}\text { RCT crossover } \\
\text { double-blind }\end{array}$ & $\begin{array}{l}\text { Dronabinol } 10 \mathrm{mg} \\
\text { or } 20 \mathrm{mg} \text { or } \\
\text { Marijuana } 1.98 \% \\
\text { or } 3.56 \% \text { THC; } \\
\text { single dose }\end{array}$ & $\begin{array}{l}\text { Changes in pain } \\
\text { tolerance }\end{array}$ & CPT & $\begin{array}{l}\text { Both marijuana and dronabinol } \\
\text { reduced pain, dronabinol } \\
\text { had longer-lasting decreases } \\
\text { in pain and lower ratings of } \\
\text { abuse-related effects than } \\
\text { marijuana }\end{array}$ & None \\
\hline $\begin{array}{l}\text { Malik et al. } \\
2017[28]\end{array}$ & $\begin{array}{l}\text { Non cardiac } \\
\text { chest pain } \\
\text { patients }(n=13)\end{array}$ & $\begin{array}{l}\text { RCT parallel } \\
\text { double-blind }\end{array}$ & $\begin{array}{l}\text { Dronabinol } \\
10 \mathrm{mg} / \text { day; } \\
28 \text { days }\end{array}$ & $\begin{array}{l}\text { Increase in pain } \\
\text { threshold and } \\
\text { reduction of } \\
\text { frequency and } \\
\text { intensity of pain }\end{array}$ & $\begin{array}{l}\text { Daily chest pain diary, } \\
\text { chest pain symptom } \\
\text { questionnaire, SF-36, } \\
\text { BAl, BDI }\end{array}$ & $\begin{array}{l}\text { Pain intensity, odynophagia, } \\
\text { and chest pain episodes } \\
\text { frequency were decreased }\end{array}$ & None \\
\hline $\begin{array}{l}\text { NCT01598207 } \\
2017[29]\end{array}$ & $\begin{array}{l}\text { Patients with } \\
\text { non-GERD } \\
\text { related non- } \\
\text { cardiac chest } \\
\text { pain }(\mathrm{n}=12)\end{array}$ & $\begin{array}{l}\text { RCT parallel } \\
\text { double-blind }\end{array}$ & $\begin{array}{l}\text { Dronabinol } \\
5 \mathrm{mg} / \text { day; } 28 \text { days }\end{array}$ & $\begin{array}{l}\text { Frequency of chest } \\
\text { pain episodes }\end{array}$ & $\begin{array}{l}\text { Frequency and } \\
\text { intensity of chest pain } \\
\text { episodes }\end{array}$ & $\begin{array}{l}\text { Dronabinol reduced the } \\
\text { intensity and duration of chest } \\
\text { pain episodes, dronabinol did } \\
\text { not significantly affect basic } \\
\text { metabolic components }\end{array}$ & None \\
\hline
\end{tabular}


Table 2. Summary of included studies: Therapeutic potential of dronabinol (cont.).

\begin{tabular}{|c|c|c|c|c|c|c|c|}
\hline $\begin{array}{l}\text { Name/author } \\
\text { and year of } \\
\text { publication }\end{array}$ & Population & Study design & $\begin{array}{l}\text { Drug intervention } \\
\text { and scheme }\end{array}$ & Primary outcome & Measures & Effects & $\begin{array}{l}\text { Adverse effects, } \\
\text { side effects, and } \\
\text { toxicity }\end{array}$ \\
\hline $\begin{array}{l}\text { Schimrigk et } \\
\text { al. } 2017[31]\end{array}$ & $\begin{array}{l}\text { MS patients } \\
\text { with central } \\
\text { neuropathic } \\
\text { pain (RCT } n= \\
\text { 238; open-label } \\
\text { CT } n=169)\end{array}$ & $\begin{array}{l}\text { RCT parallel } \\
\text { double- } \\
\text { blind, open- } \\
\text { label CT }\end{array}$ & $\begin{array}{l}\text { Dronabinol } 7.5- \\
15 \mathrm{mg} / \text { day; } \mathrm{RCT} \\
16 \text { weeks, open- } \\
\text { label } 32 \text { weeks }\end{array}$ & $\begin{array}{l}\text { Change of pain } \\
\text { intensity and safety } \\
\text { of dronabinol use }\end{array}$ & $\begin{array}{l}\text { NRS pain, SF-36, vital } \\
\text { signs, laboratory } \\
\text { parameters, ECG, case } \\
\text { report form }\end{array}$ & $\begin{array}{l}\text { Both placebo and dronabinol } \\
\text { decreased pain sensation, } \\
\text { dronabinol had a good } \\
\text { tolerability }\end{array}$ & $\begin{array}{l}\text { AEs mild to } \\
\text { moderate (e.g. } \\
\text { dizziness, vertigo, } \\
\text { fatigue), SAEs and } \\
\text { SARs rare }\end{array}$ \\
\hline $\begin{array}{l}\text { Vries et al. } \\
2017[32]\end{array}$ & $\begin{array}{l}\text { Chronic } \\
\text { pancreatitis } \\
\text { patients }(n=24)\end{array}$ & $\begin{array}{l}\text { RCT crossover } \\
\text { double-blind }\end{array}$ & $\begin{array}{l}\text { Namisol } 8 \mathrm{mg} \\
\text { single dose }\end{array}$ & $\begin{array}{l}\text { Analgesic efficacy } \\
\text { and safety }\end{array}$ & $\begin{array}{l}\text { VAS pain, vital } \\
\text { functions, SwayStar, } \\
\mathrm{ECG}, \mathrm{HR}, \mathrm{BP} \text {, } \\
\text { laboratory parameters }\end{array}$ & $\begin{array}{l}\text { Dronabinol increased anxiety } \\
\text { and } \mathrm{HR}\end{array}$ & $\begin{array}{l}\text { Mild to moderate } \\
\text { (somnolence, dry } \\
\text { mouth, dizziness, } \\
\text { euphoric mood) }\end{array}$ \\
\hline $\begin{array}{l}\text { Vries et al. } \\
2017 \text { [33] }\end{array}$ & $\begin{array}{l}\text { Chronic } \\
\text { pancreatitis } \\
\text { patients }(n=62)\end{array}$ & $\begin{array}{l}\text { RCT parallel } \\
\text { double-blind }\end{array}$ & $\begin{array}{l}\text { Namisol } 5 \text { or } \\
9 \text { mg/day; } 5 \text { days }\end{array}$ & $\begin{array}{l}\text { Regulate negative } \\
\text { affect }\end{array}$ & $\begin{array}{l}\text { VAS pain, pain-related } \\
\text { questionnaires, SF-36, } \\
\text { laboratory analysis }\end{array}$ & None & Mild to moderate \\
\hline $\begin{array}{l}\text { Amerongen et } \\
\text { al. } 2018 \text { [34] }\end{array}$ & $\begin{array}{l}\text { Healthy patients } \\
(\mathrm{n}=23)\end{array}$ & $\begin{array}{l}\text { RCT crossover } \\
\text { double-blind }\end{array}$ & $\begin{array}{l}\text { Namisol } 10 \mathrm{mg} \\
\text { or Paracetamol } \\
1000 \mathrm{mg} \text { or } \\
\text { Promethazine } \\
50 \mathrm{mg} \text {; single } \\
\text { dose }\end{array}$ & Analgesic efficacy & $\begin{array}{l}\text { eVAS, VAS: pain, } \\
\text { subjective assessment } \\
\text { of sedation and } \\
\text { psychotomimetic } \\
\text { effects }\end{array}$ & $\begin{array}{l}\text { Dronabinol increased pain } \\
\text { sensation and calmness, } \\
\text { decreased alertness and } \\
\text { perception }\end{array}$ & $\begin{array}{l}\text { Mild to moderate } \\
\text { (dizziness, } \\
\text { headache, } \\
\text { euphoric mood, } \\
\text { mild auditory } \\
\text { hallucinations) }\end{array}$ \\
\hline $\begin{array}{l}\text { Babalonis et } \\
\text { al. } 2019[35]\end{array}$ & $\begin{array}{l}\text { Healthy patients } \\
(\mathrm{n}=10)\end{array}$ & $\begin{array}{l}\text { RCT crossover } \\
\text { double-blind }\end{array}$ & $\begin{array}{l}\text { Dronabinol } \\
2.5 \mathrm{mg} \text { and } 5 \mathrm{mg} \\
\text { or Oxycodone } \\
5 \mathrm{mg} \text { or } 10 / \\
\text { session; } \\
9 \text { sessions }\end{array}$ & $\begin{array}{l}\text { Enhancement of } \\
\text { opioid analgesia }\end{array}$ & $\begin{array}{l}\text { Physiological } \\
\text { measures, DSST, VAS: } \\
\text { pain, the Participant- } \\
\text { Rated Opioid } \\
\text { Adjective Scale, the } \\
\text { Marijuana Drug Effect } \\
\text { Questionnaire }\end{array}$ & $\begin{array}{l}\text { Addictive dronabinol did not } \\
\text { increase the analgesic effects } \\
\text { of oxycodone }\end{array}$ & None \\
\hline $\begin{array}{l}\text { NCT03604341 } \\
2020[36]\end{array}$ & $\begin{array}{l}\text { Patients } \\
\text { consented for } \\
\text { elective medical } \\
\text { abortion }(n=70)\end{array}$ & $\begin{array}{l}\text { RCT parallel } \\
\text { double-blind }\end{array}$ & $\begin{array}{l}\text { Dronabinol: } \\
5 \mathrm{mg} / \text { day; } \\
10 \text { weeks }\end{array}$ & $\begin{array}{l}\text { Maximum self- } \\
\text { reported pain }\end{array}$ & NRS pain & None & $\begin{array}{l}\text { No serious AEs: } \\
\text { nausea }\end{array}$ \\
\hline \multicolumn{8}{|l|}{ Brain functions } \\
\hline $\begin{array}{l}\text { Gorka et al. } \\
2016[40]\end{array}$ & $\begin{array}{l}\text { Healthy patients } \\
(\mathrm{n}=78)\end{array}$ & $\begin{array}{l}\text { RCT parallel } \\
\text { double-blind }\end{array}$ & $\begin{array}{l}\text { Marinol } 7.5 \mathrm{mg} \\
\text { single dose }\end{array}$ & $\begin{array}{l}\text { Modulation of } \\
\text { fronto-limbic } \\
\text { activation and } \\
\text { functional } \\
\text { connectivity during } \\
\text { a volitional emotion } \\
\text { regulation task }\end{array}$ & $\begin{array}{l}\text { Subjective affection } \\
\text { ratings, fMRI: BOLD, } \\
\text { gPPI }\end{array}$ & $\begin{array}{l}\text { Dronabinol increased } \\
\text { amygdala activation and } \\
\text { reduced amygdala dorsolateral } \\
\text { prefrontal cortex functional } \\
\text { connection during cognitive } \\
\text { reappraisal }\end{array}$ & None \\
\hline $\begin{array}{l}\text { Doss et al. } \\
2018[41]\end{array}$ & $\begin{array}{l}\text { Healthy patients } \\
(\mathrm{n}=23)\end{array}$ & $\begin{array}{l}\text { RCT crossover } \\
\text { double-blind }\end{array}$ & $\begin{array}{l}\text { Marinol } 15 \mathrm{mg} \\
\text { single dose }\end{array}$ & $\begin{array}{l}\text { Changes in neural } \\
\text { and emotional } \\
\text { memories }\end{array}$ & $\begin{array}{l}\text { Emotional stimuli and } \\
\text { DRM stimuli }\end{array}$ & $\begin{array}{l}\text { Dronabinol had adverse effects } \\
\text { during memory retrieval, } \\
\text { distortion of both neutral and } \\
\text { emotional memories }\end{array}$ & \\
\hline $\begin{array}{l}\text { Rabinak et al. } \\
2018[42]\end{array}$ & $\begin{array}{l}\text { Healthy patients } \\
(\mathrm{n}=77)\end{array}$ & $\begin{array}{l}\text { RCT parallel } \\
\text { double-blind }\end{array}$ & $\begin{array}{l}\text { Marinol } 7.5 \text { mg; } \\
\text { single dose }\end{array}$ & $\begin{array}{l}\text { Changes in next- } \\
\text { day extinction } \\
\text { recall mediated by } \\
\text { post-extinction } \\
\text { resting-state brain } \\
\text { dynamics }\end{array}$ & $\begin{array}{l}\text { Subjective Drug } \\
\text { Effects, fMRI: BOLD, } \\
\text { static and dynamic } \\
\text { RS-FC, SCR, subjective } \\
\text { ratings of US } \\
\text { expectancy }\end{array}$ & $\begin{array}{l}\text { Dronabinol affected both static } \\
\text { and dynamic RS-FC within } \\
\text { fear-extinction neural circuitry } \\
\text { during a rest period following } \\
\text { extinction learning }\end{array}$ & None \\
\hline $\begin{array}{l}\text { Gilman et al. } \\
2019 \text { [44] }\end{array}$ & $\begin{array}{l}\text { Cannabis users } \\
(\mathrm{n}=54)\end{array}$ & $\begin{array}{l}\text { RCT crossover } \\
\text { double-blind }\end{array}$ & $\begin{array}{l}\text { Dronabinol } \\
5-50 \mathrm{mg} ; \text { single } \\
\text { dose }\end{array}$ & $\begin{array}{l}\text { Increased } \\
\text { prefrontal } \\
\text { activation }\end{array}$ & DEQ, HR, fNIRS & $\begin{array}{l}\text { Dronabinol increased: ratings } \\
\text { of feeling "high", HR, HbO } \\
\text { concentration throughout } \\
\text { the prefrontal cortex, and } \\
\text { concentration degradation in } \\
\text { the cognitive task }\end{array}$ & $\begin{array}{l}\text { Vomiting, } \\
\text { nausea, paranoia, } \\
\text { sedation, } \\
\text { tachycardia, } \\
\text { anxiety, } \\
\text { dizziness, } \\
\text { bradycardia, } \\
\text { syncope }\end{array}$ \\
\hline $\begin{array}{l}\text { Hammoud et } \\
\text { al. } 2019[46]\end{array}$ & $\begin{array}{l}\text { Healthy patients } \\
(n=40)\end{array}$ & $\begin{array}{l}\text { RCT parallel } \\
\text { double-blind }\end{array}$ & $\begin{array}{l}\text { Dronabinol } \\
7.5 \mathrm{mg} \text {; single } \\
\text { dose }\end{array}$ & $\begin{array}{l}\text { Impact on } \\
\text { functional } \\
\text { activation of the } \\
\text { threat extinction } \\
\text { network }\end{array}$ & $\begin{array}{l}\text { SCR, fMRI: gPPI and } \\
\text { BOLD }\end{array}$ & $\begin{array}{l}1 \text { week after learning } \\
\text { dronabinol decreased } \\
\text { responses within the } \\
\text { ventromedial prefrontal cortex } \\
\text { and amygdala but increased } \\
\text { responses within the threat } \\
\text { extinction network during the } \\
\text { extinction retention test }\end{array}$ & None \\
\hline
\end{tabular}


Table 2. Summary of included studies: Therapeutic potential of dronabinol (cont.).

\begin{tabular}{|c|c|c|c|c|c|c|c|}
\hline $\begin{array}{l}\text { Name/author } \\
\text { and year of } \\
\text { publication }\end{array}$ & Population & Study design & $\begin{array}{l}\text { Drug intervention } \\
\text { and scheme }\end{array}$ & Primary outcome & Measures & Effects & $\begin{array}{l}\text { Adverse effects, } \\
\text { side effects, and } \\
\text { toxicity }\end{array}$ \\
\hline \multicolumn{8}{|c|}{ Obstructive Sleep Apnea } \\
\hline $\begin{array}{l}\text { NCT01755091 } \\
2017[48]\end{array}$ & $\begin{array}{l}\text { Patients with } \\
\text { OSA }(n=56)\end{array}$ & $\begin{array}{l}\text { RCT parallel } \\
\text { triple-blind }\end{array}$ & $\begin{array}{l}\text { Dronabinol: } \\
2.5 \mathrm{mg} \text { or } \\
10 \mathrm{mg} / \mathrm{day} ; \\
6 \text { weeks }\end{array}$ & $\begin{array}{l}\text { Change in AHI, ESS, } \\
\text { and sleep latency }\end{array}$ & AHI, ESS, MWT & $\begin{array}{l}\text { Dronabinol decreased AHI, } \\
\text { improved self-reported } \\
\text { sleepiness, and had } \\
\text { great treatment } \\
\text { satisfaction }\end{array}$ & $\begin{array}{l}\text { A little serious } \\
\text { AEs: nausea, } \\
\text { headache }\end{array}$ \\
\hline
\end{tabular}

\section{Nausea and vomiting}

\begin{tabular}{|c|c|c|c|c|c|c|c|}
\hline $\begin{array}{l}\text { NCT00757822 } \\
2016[50]\end{array}$ & $\begin{array}{l}\text { Patients being } \\
\text { undergone } \\
\text { surgery }(n=121)\end{array}$ & $\begin{array}{l}\text { RCT parallel } \\
\text { triple-blind }\end{array}$ & $\begin{array}{l}\text { Dronabinol } 5 \mathrm{mg} \\
\text { or Ondansetron } \\
4 \mathrm{mg} \text {; single dose }\end{array}$ & $\begin{array}{l}\text { Incidence of } \\
\text { postoperative } \\
\text { nausea and } \\
\text { vomiting }\end{array}$ & $\begin{array}{l}\text { PACU, VAS nausea, } \\
\text { PONV }\end{array}$ & None & None \\
\hline \multicolumn{8}{|c|}{ Tourette Syndrome } \\
\hline $\begin{array}{l}\text { NCT03066193 } \\
2019[52]\end{array}$ & $\begin{array}{l}\text { Patients with TS } \\
(\mathrm{n}=16)\end{array}$ & $\begin{array}{l}\text { Open-label } \\
\text { clinical trial }\end{array}$ & $\begin{array}{l}\text { Dronabinol } 2.5- \\
10 \mathrm{mg} \text { and PEA } \\
400 \mathrm{mg} / \mathrm{day} ; \\
12 \text { weeks }\end{array}$ & $\begin{array}{l}\text { Changes in tic } \\
\text { severity }\end{array}$ & YGTSS & $\begin{array}{l}\text { The combination of dronabinol } \\
\text { and PEA reduced tic severity in } \\
\text { TS during therapy }\end{array}$ & $\begin{array}{l}\text { No serious AEs: } \\
\text { fatigue, feeling } \\
\text { "high", dizziness } \\
\text { difficulty } \\
\text { concentrating, } \\
\text { and dry mouth }\end{array}$ \\
\hline
\end{tabular}

\begin{tabular}{|c|c|c|c|c|c|c|c|}
\hline \multicolumn{8}{|c|}{ Relieving stress } \\
\hline $\begin{array}{l}\text { Childs et al. } \\
2017 \text { [53] }\end{array}$ & $\begin{array}{l}\text { Healthy patients } \\
(n=42)\end{array}$ & $\begin{array}{l}\text { RCT parallel } \\
\text { double-blind }\end{array}$ & $\begin{array}{l}\text { Dronabinol } \\
7.5 \mathrm{mg} \text { or } \\
12.5 \mathrm{mg} / \mathrm{session} ; \\
2 \text { sessions }\end{array}$ & $\begin{array}{l}\text { Changes in self- } \\
\text { reported subjective } \\
\text { distress }\end{array}$ & $\begin{array}{l}\text { TSST, non-stressful } \\
\text { task, PASA VAS, } \\
\text { HR, BP, MAP, ARCI, } \\
\text { DEQ, POMS, salivary } \\
\text { cortisol }\end{array}$ & $\begin{array}{l}\text { The low dose of dronabinol } \\
\text { reduced subjective distress, } \\
\text { but higher doses increased } \\
\text { negative mood }\end{array}$ & None \\
\hline
\end{tabular}

\begin{tabular}{|c|c|c|c|c|}
\hline Anorexia & & & & \\
\hline $\begin{array}{l}\text { Weltens et al. } \\
2019[54]\end{array}$ & $\begin{array}{l}\text { Healthy patients } \\
(n=17)\end{array}$ & $\begin{array}{l}\text { RCT crossover } \\
\text { single-blind }\end{array}$ & $\begin{array}{l}\text { Dronabinol } \\
0.1 \mathrm{mg} / \mathrm{kg} / \text { sessio; } \\
4 \text { sessions }\end{array}$ & $\begin{array}{l}\text { Changes in food } \\
\text { intake and hunger }\end{array}$ \\
\hline
\end{tabular}

$\begin{array}{lll}\text { VAS food wanting } & \text { Dronabinol increased: liking } & \text { Commonly: } \\ \text { and liking, food } & \text { and wanting of the high- } & \text { feeling, ,high”, } \\ \text { intake, blood samples } & \text { calorie images, food intake, } & \text { dizzy, sleepy and } \\ \text { (plasma octanoylated } & \text { plasma motilin, and decreased } & \text { sad, headache, } \\ \text { ghrelin, motilin, } & \text { octanoylated ghrelin } & \text { dry mouth, sense } \\ \text { GLP-1) } & \text { concentrations } & \text { of unreality }\end{array}$

\begin{tabular}{|c|c|c|c|c|c|c|c|}
\hline \multicolumn{8}{|c|}{ Dementia neuropsychiatric symptoms } \\
\hline $\begin{array}{l}\text { van del Elsen } \\
\text { et al. } 2017[56]\end{array}$ & $\begin{array}{l}\text { Dementia } \\
\text { patients }(n=18)\end{array}$ & $\begin{array}{l}\text { RCT crossover } \\
\text { double-blind }\end{array}$ & $\begin{array}{l}\text { Namisol } 3 \text { mg; } \\
3 \text { days }\end{array}$ & $\begin{array}{l}\text { Changes in balance } \\
\text { and gait }\end{array}$ & $\begin{array}{l}\text { SwayStar system, } \\
\text { GAITRite system, } \\
\text { subjective mobility } \\
\text { tasks }\end{array}$ & $\begin{array}{l}\text { Dronabinol worsened the } \\
\text { balance and disturbed the gait }\end{array}$ & None \\
\hline
\end{tabular}

\begin{tabular}{|c|c|c|c|c|c|c|c|}
\hline \multicolumn{8}{|l|}{ Abuse potential } \\
\hline $\begin{array}{l}\text { Schoedel et al. } \\
2018 \text { [57] }\end{array}$ & $\begin{array}{l}\text { Healthy patients } \\
(n=43)\end{array}$ & $\begin{array}{l}\text { RCT crossover } \\
\text { double-blind }\end{array}$ & $\begin{array}{l}\text { Dronabinol } \\
10 \text { or } 30 \mathrm{mg} \\
\text { or CBD } 750 \text { or } \\
1500 \text { or } 4500 \mathrm{mg} \\
\text { or Alprazolam } \\
2 \mathrm{mg} / \text { session; } \\
7 \text { sessions }\end{array}$ & $\begin{array}{l}\text { The abuse potential } \\
\text { of dronabinol }\end{array}$ & $\begin{array}{l}\text { DAT, HVLT-R, DSST, } \\
\text { blood samples, VAS: } \\
\text { Overall Drug-Liking, } \\
\text { Take Drug Again, Drug } \\
\text { Similarity }\end{array}$ & $\begin{array}{l}\text { Dronabinol had higher } \\
\text { abuse potential than CBD } \\
\text { and placebo, greater dose } \\
\text { was related to higher abuse } \\
\text { potential }\end{array}$ & $\begin{array}{l}\text { No serious AEs: } \\
\text { dry mouth, } \\
\text { euphoric mood } \\
\text { somnolence, } \\
\text { nausea }\end{array}$ \\
\hline \multicolumn{8}{|l|}{ Hemodynamics } \\
\hline $\begin{array}{l}\text { Hommer et al. } \\
2020 \text { [58] }\end{array}$ & $\begin{array}{l}\text { Healthy patients } \\
(\mathrm{n}=24)\end{array}$ & $\begin{array}{l}\text { RCT crossover } \\
\text { double-blind }\end{array}$ & $\begin{array}{l}\text { Dronabinol } 5 \mathrm{mg} ; \\
\text { single dose }\end{array}$ & $\begin{array}{l}\text { Increase of optic } \\
\text { nerve head blood } \\
\text { flow }\end{array}$ & $\begin{array}{l}\text { ONHBF, OPP IOP, MAP, } \\
B P, H R\end{array}$ & Dronabinol increased ONHBF & None \\
\hline
\end{tabular}

Abbreviations: RTC, Randomized Controlled Trial; TLFB, Timeline Follow Back; WDS, Withdrawal Discomfort Score; MCQ, Marijuana, Craving Questionnaire; AE, Adverse Effect; MWC, Marijuana Withdrawal Checklist; VAS, Visual Analog Scale; HR, Heart Rate; fMRI, Functional Magnetic Resonance Imaging; SOWS, Short Opiate Withdrawal Scale; CPT, Cold-Pressor Test; 00WS, Objective Opiate Withdrawal Scale; SF-36, Short Form 36 Health Survey Questionnaire; BAI, Beck Anxiety Inventory; BDI, Beck Depression Inventory; GERD, Gastroesophageal Reflux Disease; MS, Multiple Sclerosis; NRS, Numeric Rating Scale; ECG, Electrocardiography; SAE, Serious Adverse Effect; SAR, Serious Adverse Reaction; BP, Blood Pressure; DSST, Digit Symbol Substitution Task; BOLD, Blood Oxygenation Level Dependent; gPPI, Generalized Psychophysiological Interaction Analysis; DRM, Deese-Roediger-McDermott Paradigm; RS-FC, Resting-State Functional Connectivity; DEQ, Drug Effects Questionnaire; fNIRS, Functional Near Infrared Spectroscopy; HbO, Oxyhemoglobin; SCR, Skin Conductance Response; OSA, Obstructive Sleep Apnea; AHI, Apnea/Hypopnea Index; ESS, Epworth Sleepiness Scale; MWT, Maintenance of Wakefulness Test; PACU, Post-Operative Care Unit; PONV, Post-Operative Nausea and Vomiting; TS, Tourette Syndrome; PEA, Palmitoylethanolamide; YGTSS, Yale Global Tic Severity Scale; TSST, Trier Social Stress Test; PASA VAS, post-task appraisal questionnaire VAS; ARCI, Addiction Research Center Inventory; POMS, Profile of Mood States; GLP-1, Glucagon-Like Peptide 1; CBD, Cannabidiol; DAT, Divided Attention Test; HVLT, Hopkins Verbal Learning Test-Revised; ONHBF, Optic Nerve; Head Blood Flow; OPP, Ocular Perfusion Pressure; IOP, Intraocular Pressure; MAP, Mean Arterial Blood Pressure 


\section{Limitations of review}

The main limitation is the small size of the study groups in numerous included studies. That's why obtained outcomes might not correspond to the results if the cohort was greater, which significantly reduces the credibility of included articles. Only trials published in English were included, excluding potentially relevant trials published in other languages. Another limitation is the design of included studies. There was no placebo group in open-label clinical trials, which prevents the authors from fully determining the therapeutic effect of the implemented drug. In addition, most of the studies were of short-term duration, so long-term administration effects cannot be concluded. Only one study lasted 32 weeks, while some of the reviewed articles analyzed only a single dose of dronabinol.

\section{The risk of bias within studies}

Based on the GRADE, we evaluated the certainty of reviewed articles. The evaluation results exhibited that the overall certainty of registered clinical trials was very low to moderate, indicating that most registered studies had a great risk of bias. The main reasons for the low certainty were: imprecision, attrition bias, and inconsistency. The certainty of reviewed studies is presented in Table 3.

\section{Dronabinol in cannabis use disorder treatment}

Levine et al. in a double-blind, parallel RCT published in 2016 investigated the impact of the concurrent administration of lofexidine and dronabinol on promoting abstinence and reducing withdrawal symptoms among 122 cannabis-dependent adults. Results of a trial did not show any effect of a treatment on 21-days consecutive abstinence and withdrawal scores across time. The authors indicated that the pharmacotherapy did not promote abstinence, but may have an effect on relapse prevention. In order to investigate it in future studies, detoxification should be started before randomization. Study AEs

Table 3. Certainty of included studies: Therapeutic potential of dronabilon.

\begin{tabular}{|c|c|c|c|}
\hline Outcome & $\begin{array}{l}\text { Number of participants } \\
\text { (studies) }\end{array}$ & $\begin{array}{l}\text { Certainty of the } \\
\text { evidence (GRADE) }\end{array}$ & Effect \\
\hline $\begin{array}{l}\text { Dronabinol has potential in cannabis use disorder } \\
\text { therapy in high daily dose administration }\end{array}$ & 178 (6 RCTs) & $\begin{array}{l}\oplus \oplus \oplus \bigcirc \\
\text { moderate }\end{array}$ & $\begin{array}{l}\text { Four of six studies showed a positive effect of dronabinol in } \\
\text { cannabis use disorder therapy in comparison to placebo }\end{array}$ \\
\hline $\begin{array}{l}\text { Dronabinol has no potential in opioid addiction } \\
\text { therapy }\end{array}$ & $12(1 \mathrm{RCT})$ & $\begin{array}{l}\oplus \bigcirc \bigcirc \bigcirc \\
\text { very low }\end{array}$ & $\begin{array}{l}\text { The study did not show a positive effect of dronabinol in opioid } \\
\text { addiction treatment in comparison to placebo and other } \\
\text { medication }\end{array}$ \\
\hline Dronabinol has low potential to be an analgesics & 482 (9 RCTs) & $\begin{array}{l}\oplus \oplus \oplus \bigcirc \\
\text { moderate }\end{array}$ & $\begin{array}{l}\text { Six of nine studies showed no positive effect of dronabinol } \\
\text { pharmacotherapy in pain management in comparison to } \\
\text { placebo }\end{array}$ \\
\hline $\begin{array}{l}\text { Dronabinol caused dysregulation of particular brain } \\
\text { areas }\end{array}$ & 272 (5 RCTs) & $\begin{array}{l}\oplus \oplus \oplus \bigcirc \\
\text { moderate }\end{array}$ & $\begin{array}{l}\text { Four of five studies showed the degradation effect of dronabinol } \\
\text { on cognitive tasks and one showed that PFC activation can be } \\
\text { a potential biomarker for THC intoxication }\end{array}$ \\
\hline $\begin{array}{l}\text { Dronabinol has potential in OSA symptoms } \\
\text { treatment }\end{array}$ & $56(1 \mathrm{RCT})$ & $\begin{array}{l}\oplus \oplus \circ \bigcirc \\
\text { low }\end{array}$ & $\begin{array}{l}\text { The study showed a positive effect of dronabinol in OSA } \\
\text { symptoms treatment in comparison to placebo }\end{array}$ \\
\hline $\begin{array}{l}\text { Dronabinol has less potential in nausea and } \\
\text { vomiting treatment in comparison to Ondansetron }\end{array}$ & $121(1 \mathrm{RCT})$ & $\begin{array}{l}\oplus \oplus \bigcirc \bigcirc \\
\text { low }\end{array}$ & $\begin{array}{l}\text { The study did not show a positive effect of dronabinol in nausea } \\
\text { and vomiting treatment in comparison to other medication }\end{array}$ \\
\hline $\begin{array}{l}\text { The combination of PEA and dronabinol has } \\
\text { potential in TS symptoms treatment }\end{array}$ & $\begin{array}{l}16 \text { ( } 1 \text { open-label clinical } \\
\text { trial) }\end{array}$ & $\begin{array}{l}\oplus \bigcirc \bigcirc \bigcirc \\
\text { very low }\end{array}$ & $\begin{array}{l}\text { The study showed a positive effect of the combination of } \\
\text { dronabinol and PEA in TS symptoms treatment }\end{array}$ \\
\hline $\begin{array}{l}\text { Dronabinol low dosage administration has the } \\
\text { potential in relieving stress }\end{array}$ & $42(1 \mathrm{RCT})$ & $\begin{array}{l}\oplus \oplus \bigcirc \bigcirc \\
\text { low }\end{array}$ & $\begin{array}{l}\text { The study showed a positive effect of the low dose of dronabinol } \\
\text { in relieving stress in comparison to the placebo }\end{array}$ \\
\hline $\begin{array}{l}\text { Dronabinol has potential in appetite and food intake } \\
\text { increasing }\end{array}$ & $17(1 \mathrm{RCT})$ & $\begin{array}{l}\oplus \bigcirc \bigcirc \bigcirc \\
\text { very low }\end{array}$ & $\begin{array}{l}\text { The study showed a positive effect of dronabinol on appetite } \\
\text { and food intake in comparison to placebo }\end{array}$ \\
\hline $\begin{array}{l}\text { Dronabinol has no potential in dementia } \\
\text { neuropsychiatric symptoms treatment }\end{array}$ & $18(1 \mathrm{RCT})$ & $\begin{array}{l}\oplus \bigcirc \bigcirc \bigcirc \\
\text { very low }\end{array}$ & $\begin{array}{l}\text { The study showed no positive effect of dronabinol in dementia } \\
\text { neuropsychiatric symptoms treatment in comparison to } \\
\text { placebo }\end{array}$ \\
\hline $\begin{array}{l}\text { Dronabinol has greater abuse potential in } \\
\text { comparison to CBD, its abuse potential is related } \\
\text { to dosage }\end{array}$ & $43(1 \mathrm{RCT})$ & $\begin{array}{l}\oplus \oplus \bigcirc \bigcirc \\
\text { low }\end{array}$ & $\begin{array}{l}\text { The study showed higher abuse potential of dronabinol than CBD } \\
\text { and placebo, greater dose was related to higher abuse potential }\end{array}$ \\
\hline Dronabinol increase ocular blood flow & $24(1 \mathrm{RCT})$ & $\begin{array}{l}\oplus \oplus \bigcirc \bigcirc \\
\text { low }\end{array}$ & The study showed an increase in ocular blood flow \\
\hline
\end{tabular}

Abbreviations: GRADE, Grading of Recommendation. Assessment, Development and Evaluation; RCT, Randomized Controlled Trial; OSA, Obstructive Sleep Apnea; PEA, Palmitoylethanolamide; TS, Tourette Syndrome; CBD, cannabidiol. Symbols are used to describe certainty in evidence profiles. High certainty: $\oplus \oplus \oplus \oplus$; Moderate certainty: $\oplus \oplus \oplus \bigcirc$; Low certainty: $\oplus \oplus \bigcirc \bigcirc$; Very low certainty: $\oplus \bigcirc \bigcirc \bigcirc$. 
included dry mouth, intoxication, and hypotension and only two of them were serious [13].

A double-blind, parallel RCT analyzed the impact of dronabinol on marijuana smoking cessation. The clinical trial was published in 2017 and performed on 13 cannabis smokers. Dronabinol reduced the number of cannabis cigarettes smoked during therapy in comparison to placebo. There was no difference between the high and the low dosage of administered medication. There were no AEs reported [14].

Published in 2017 and performed on 13 patients with cannabis use disorder diagnosed, a double-blind, crossover RCT analyzed the dronabinol potential in smoking cessation. In the study, dronabinol decreased cannabis self-administration and suppressed withdrawal symptoms. There were no serious adverse events (SAEs), no major events included: dry mouth and anxiety $[15,16]$.

Results of published in 2018 a parallel, quadruple-blinded RCT investigated the potential of a combination of dronabinol and clonidine in cannabis use disorder. The study was performed on five marijuana smokers with schizophrenia. The concurrent pharmacotherapy of dronabinol and clonidine reduced the number of cannabis cigarettes smoked during therapy in comparison to placebo. No AEs were registered [17].

The impact of dronabinol on cannabis use disorder treatment was also developed in a double-blind, parallel RCT $(\mathrm{n}=12)$ published in 2019. The authors analyzed physiological changes in response to cue-induced craving and tolerability of treatment. Dronabinol administration during marijuana abstinence decreased ratings of anxiety, misery, sleeping troubles, chills, marijuana craving, and increased food intake in comparison to placebo. There were no SAEs, while minor events included: drowsiness and increased craving [18, 19].

Schlienz et al. published results of 12-days double-blind, crossover RCT with 13 cannabis users. The patients received dronabinol in doses: 120 or $180-240 \mathrm{mg}$ daily. The results of this trial yielded the following results: maintenance on both low-dose and high-dose dronabinol significantly reduced self-administration of cannabis compared with placebo. Dronabinol therapy significantly reduced cannabis withdrawal ravages both in self-report (e.g decreased appetite, irritability, sweating, depressed mood, restlessness, sleep difficulty, increased aggression, stomach pains) and the Withdrawal Discomfort Score. Low-dose dronabinol reduced both systolic and diastolic blood pressure (BP) while high-dose influenced additionally the heart rate (HR). AEs were not observed [20].

Estimated to be finished in 2022 an open-label clinical trial will evaluate the dronabinol pharmacotherapy (10-60 mg) effects on sleep among frequent cannabis users and non-frequent cannabis users. This pilot study will be performed on healthy young adults without a history of chronic disease [21].

Another study evaluating the dronabinol potential in cannabis use disorder treatment is a quadruple, parallel RCT. It is estimated to be completed in 2021. The study will emphasize on Activation of the Brain Reward Circuit (particularly the nucleus accumbens) in anticipation of monetary reward [22].

\section{Dronabinol as opioid addiction therapeutic option}

The effect of dronabinol was studied in 12 opioid-dependent patients during a double-blind, parallel RCT. The drug was administered at doses: $5,10,20$, or $30 \mathrm{mg}$ alternating with Oxycodone at doses: 30 or $60 \mathrm{mg}$ per session. It was found that dronabinol in high quantities produced rates of "good effects", "bad effects" and "sedation" and modestly decreased desire for opiates in Visual Analog Scale (VAS). Moreover, it lessened cognitive measures the most, did not differentiate from placebo in opioid agonist and antagonist adjective scale, and increased HR causing tachycardia and nervousness. To summarize, dronabinol is not a likely candidate for the treatment of opioid withdrawal [23].

In 2019 was scheduled termination of a 12-week open-label clinical trial, performed on 280 patients with chronic pain. Participants will be treated with up to $20 \mathrm{mg}$ of Dronabinol per day in combination with previously coadministered opioid analgesic treatment. Results of the study will present an assessment of the change in pain sensation, reduction of opioid self-administration, and related side effects [24].

Another study will end in 2024. A parallel, double-blind RCT will examine $5 \mathrm{mg}$ dronabinol daily administration for 30 days decreasing opioid consumption in 460 patients undergoing total knee arthroplasty. Additionally to the quantity of Morphine Equivalence of pain medication intake, Jennings et. al. will record the nausea outcomes, Defense and Veterans Pain Scale, The Pittsburgh Sleep Quality Index, Knee Society Score, KOOS-12 Knee Survey, and The Veterans Rand 12-Item Health Survey [25]. 


\section{Dronabinol effects in pain treatment}

A double-blinded, crossover RCT analyzed the impact of dronabinol (10 or $20 \mathrm{mg}$ a day) and marijuana $(1.98 \%$ or $3.56 \%$ THC) pain tolerance using the cold pressor test (CPT). The clinical trial was published in 2017 and performed on 30 marijuana smokers. Both marijuana and dronabinol reduced pain in comparison to placebo. There was an observed decrease in pain sensitivity, increase in pain tolerance and decrease in subjective ratings of pain intensity. However, dronabinol had longer-lasting decreases in pain and lower ratings of abuse-related effects than marijuana. There were no AEs $r$ eported [26, 27].

A study conducted among 13 non-cardiac chest pain patients showed that pain intensity and odynophagia frequency were distinctly decreased after 28 days of $10 \mathrm{mg}$ a day of dronabinol treatment. Additionally, decreased frequency of chest pain episodes was observed with no treatment-associated AEs reported [28].

Results of a published in 2017 parallel, double-blinded RCT $(\mathrm{n}=12)$ investigated the potential of dronabinol in non-GERD (gastroesophageal reflux disease) related non-cardiac chest pain management. The researchers evaluated dronabinol pharmacotherapy's impact on the frequency and intensity of chest pain episodes. Dronabinol reduced the intensity and duration of chest pain episodes in comparison to placebo. Moreover, pharmacotherapy did not significantly affect basic metabolic components, so that this cannabinoid may be able to be used in patients with metabolic disorders. There were no AEs registered $[29,30]$.

A double-blind, parallel RCT with dronabinol (7.5-15 mg a day) performed on 238 multiple sclerosis (MS) patients with neuropathic pain demonstrated a decrease of mean pain intensities during 16-weeks treatment, without reaching a statistically significant difference between both groups. 169 patients fulfilled 32-weeks open-label period and 85 patients continued therapy for overall up to 119 weeks. The occurrence of severe and serious adverse reactions (SARs) was rare. Although the trial did not prove the effectiveness of the treatment, it demonstrated no signs of drug abuse and suggested that dronabinol is a safe long-term treatment option [31].

Dronabinol single dose intervention (Namisol $8 \mathrm{mg}$ ) did not prove effective in abdominal pain treatment among chronic pancreatitis patients. A double-blind, crossover RCT revealed that the pharmacokinetics parameters for dronabinol do not differ between opioid and non-opioid users and are not dependent on CYP2C9 and CYP2C19 genetic polymorphism. Moreover, dronabinol did not affect alertness, mood, calmness, and balance outcomes, but researchers indicated an increase in HR. AEs were mild to moderate and included somnolence, dry mouth, dizziness, and euphoric mood [32].

Phase 2 placebo-controlled study of Vries et al., where Namisol was administered in dosages of 5 or $9 \mathrm{mg}$ a day for 5 days confirmed an outcome of the previous clinical trial that dronabinol did not change pain perception. In addition, the authors indicated no differences in generalized anxiety and depression, change in appetite level, quality of life, treatment satisfaction, and subjective feelings. However, the evaluation of the pharmacokinetics at an individual patient level revealed that some patients demonstrate a relatively late time to reach maximum plasma concentration accompanied by a relatively low maximum concentration [33].

Another study investigating the effect profile of dronabinol (Namisol $10 \mathrm{mg}$ ) using electrical stimulation, pressure stimulation, thermal pain, and the CPT in 23 healthy subjects indicate that oral-THC decreases pain tolerance. Moreover, dronabinol increased calmness, but decreased alertness and caused a change in internal and external perception. Registered AEs were mild to moderate (dizziness, headache, euphoric mood, mild auditory hallucinations) [34].

Babalonis et al. in 2019 has observed that dronabinol in doses of 2.5 and $5 \mathrm{mg}$ did not alter the analgesic effects of oxycodone in pressure algometer, cold pressure, and hot and cold thermode tests, although increased abuse effects. No AEs were registered [35].

A double-blind, parallel RCT also analyzed the impact of dronabinol on pain management with emphasis on maximal level of experienced pain. The clinical trial was published in 2020 and performed on 70 women who consented to elective medical abortion. There was no significant effect of cannabinoid pharmacotherapy in comparison to placebo. No AEs were reported [36, 37].

The year 2023 is the estimated date of the end for two double-blind RCTs. The first one conducted on 90 patients undergoing 1 to 3 level posterolateral fusion will investigate the effect of $2.5 \mathrm{mg}$ of dronabinol versus placebo on postoperative opioid consumption [38]. Oral cannabinoids will be administered before surgery and continued twice daily for three days after. The second one will study the impact of dronabinol on post- operative reduction of pain and the need for opioid medication in 80 patients undergoing total knee replacement [39]. 


\section{Dronabinol modulation of brain function}

Single-dose of Marinol (7.5 mg) among healthy patients $(\mathrm{n}=78)$ was found to be associated with greater amygdala activation and lesser amygdala - dorsolateral prefrontal cortex (PFC) functional coupling during cognitive reappraisal of negative pictures. Therefore, the amygdala response to negative stimuli might be unregulated, creating sustained hyperactivity. Results suggest that THC affects volitional forms of emotion processing such as frontolimbic circuit during cognitive reappraisal [40].

A double-blind, crossover RCT published in 2018 evaluated the impact of dronabinol (Marinol, $15 \mathrm{mg}$ ) on brain functions - especially changes in neural and emotional memories. During tests, participants underwent an emotional memory task and a false memory task. In contrast to placebo, dronabinol pharmacotherapy had adverse effects during memory retrieval, distorted both neutral and emotional memories [41].

The study of Rabinak et al. reflected the effects of a single dose of $7.5 \mathrm{mg}$ Marinol during the possible consolidation of the extinction memory within fear-extinction neural circuitry. Moreover, the authors developed the hypothesis that synthetic cannabinoids had reduced the strength of Resting-State Functional Connectivity (RSFC) in extinction-related neural circuitry during a time period in which the consolidation of the extinction memory occurs. In a double-blind, parallel RCT among 77 healthy patients, authors proved that THC was associated with enhanced dynamic RS-FC between the amygdaloid body and ventromedial (vmPFC) and reduced static RS-FC between amygdala and hippocampus during extinction learning. Therefore, THC may help to normalize reductions in dynamic RS-FC. Recall of extinction learning was associated with higher hippocampus activation with no association between both static or dynamic post-extinction RS-FC and vmPFC activation, which gives a conclusion that THC administration enhances extinction recall. The authors did not record AEs and indicated that the study had proved the potential of dronabinol as a therapeutic option for patients with PTSD [42].

Rabinak et.al. had conducted another study, which was completed in 2019, but the study results were not published yet. This time 123 participants were diagnosed with PTSD in a parallel, double-blind RCT. The authors investigated the effects of 5 and $10 \mathrm{mg}$ of dronabinol on retention of memory for fear extinction learning. There is a high therapeutic potential of oral-THC in psychiatric conditions treatment so that the results of this study are promising [43].

A double-blind, crossover RCT published in 2019 indicated that self-reported intoxication from dronabinol (5-50 mg), which was correlated with increased HR, produced impairment of cognitive function via binding to $\mathrm{CB} 1$ receptors in the PFC. Researchers used functional Near-Infrared Spectroscopy (fNIRS) to examine the correlation between increased oxyhemoglobin $(\mathrm{HbO})$ response in the PFC and intoxication level and N-back task to analyze the change in cognitive functions. The study suggested that brain activation in this particular region could be a potential biomarker for THC intoxication. AEs were associated with dronabinol treatment and mostly included vomiting, nausea, paranoia, sedation, tachycardia, anxiety, dizziness, and bradycardia [44].

Another double-blind, crossover RCT is conducted by Evins et al.. The study completion date is scheduled for December 2020. The authors will investigate the effects of dronabinol $(\mathrm{n}=150)$ and dronabinol with ethanol $(n=50)$ among weekly or greater marijuana smokers on PFC activity with fNIRS during resting state and during memory task performance. It may confirm the conclusion of the previously mentioned study [45].

In a double-blind, parallel RCT conducted by Hammoud et al., a significant impact of THC on neural circuits of threat-extinction and the expression of this extinction memory was observed. One day study revealed that a single dose of $7.5 \mathrm{mg}$ dronabinol increased amygdala and vmPFC activation during early extinction learning with no impact on skin conductance responses. On the 10'th day of the study the experimental group presented decreased responses within the vmPFC and amygdala, but distinctly increased functional coupling in the extinction network (between the vmPFC, hippocampus, and dorsal anterior cingulate cortex) during the extinction retention test. The authors concluded that THC has a long-term impact on the functional activation of the threat extinction network [46].

The first study, which will systematically test the potential impact of $5 \mathrm{mg}$ dronabinol and $100 \mathrm{mg}$ Epidiolex on cognition in bipolar disorder is estimated to end in 2023. In triple-blind parallel RCT, several cognitive domains (arousal, decision making, cognitive control, inhibition, sense of timing) and cerebrospinal levels of anandamide and homovanillic acid (markers of endocannabinoid and dopamine activity in the brain) will be tested. An experimental group will be conducted on 144 bipolar disorder diagnosed patients [47]. 


\section{Dronabinol as pharmacotherapy of Obstructive Sleep Apnea}

A triple-blind, parallel RCT analyzed the impact of dronabinol on Apnea/Hypopnea Index (AHI), Epworth Sleepiness Scale (ESS), and sleep latency - the OSA symptoms indicators. The clinical trial was published in 2017 and performed on 56 patients with diagnosed OSA. Dronabinol decreased AHI, improved self-reported sleepiness, and had great treatment satisfaction in comparison to placebo. A few SAEs were observed, including nausea and headache. Minor AEs occurred more often in higher dose dronabinol administration (i.a. increased sleepiness, headache, nausea) [48, 49].

\section{Dronabinol for nausea and vomiting}

Published in 2016 and performed on 121 patients being undergone surgery, a triple-blind, parallel RCT analyzed the dronabinol potential in nausea and vomiting pharmacotherapy. The cannabinoid was compared to the other medication - Ondansetron with no placebo group. Overall there was no significant advantage of using dronabinol. There were some serious AEs however, it seems that none of them were caused by dronabinol administration [50].

"Multicenter, Randomized, Double-blind, Placebo-controlled, Phase III Clinical Trial to Investigate the Efficacy and Safety of Dronabinol in the Improvement of Chemotherapy-induced and Tumor-Related Symptoms in Patients With Locally Advanced or Metastatic Pancreatic Cancer During First-line Chemotherapy (DISCOVER)" is estimated to be completed in 2022. The study conducted among 140 participants will assess whether dronabinol will have an influence on the quality of life ((QLQ)-C30) and whether symptoms (including e.g. nausea, vomiting, and change in body mass) caused by the tumor or the chemotherapy will be alleviated by $0,2-30 \mathrm{mg}$ dronabinol doses [51].

\section{Dronabinol potential in Tourette Syndrome}

An open-label clinical trial analyzed the impact of a combination of dronabinol and palmitoylethanolamide (PEA) on tic severity in TS. The study was published in 2019 with a control group of 16 participants with diagnosed TS. Research showed significant improvement of tic severity after 12 weeks of pharmacotherapy. There were no SAEs but reported side effects include fatigue, feeling "high", dizziness, difficulty in concentrating, and dry mouth [52].

\section{Dronabinol for stress relief}

The study of Childs et al. presented an opposite conclusion in comparison to previously mentioned studies $[40,46]$, where a single dose of $7.5 \mathrm{mg}$ dronabinol increased amygdala activity. In a double-blind, parallel RCT the activity of the amygdaloid body was probably decreased because lower-dose of THC reduced the subjective stress and relieved tension and anxiety during both non-stressful task tests (Control) and Trier Social Stress Test (TSST). However, 12,5 mg THC elevated the post-task emotional responses during both sessions. In addition, the higher dose of dronabinol dampened the TSST-induced increase in mean arterial pressure (MAP), which changed during therapy with placebo and lower-dose groups. Dronabinol did not alter HR and salivary cortisol. No AEs were stated [53].

\section{Dronabinol effects in anorexia}

The effect of dronabinol on subjective and metabolic hormone responses to visual food stimuli and food intake was investigated in an RCT published in 2019. The main effects of the drug were increases in milkshake oral intake, liking, and wanting ratings for the high-calorie food images. No effect was observed for wanting the intragastric infusion of food. The authors indicated hormone changes. After dronabinol administration but before milkshake consumption concentration of plasma octanoylated ghrelin was significantly lower, motilin was increased, while glucagon-like peptide 1 (GLP-1) did not change. After milkshake consumption, plasma motilin and ghrelin concentrations did not change, but GLP-1 decreased. In both cases, there was no drug-by-time interaction. Dronabinol ( $0.1 \mathrm{mg} / \mathrm{kg} / \mathrm{session}) \mathrm{did}$ not impact hunger, appetite, satiety, and fullness nor any drug-by-time interaction. Among the control group, nausea, headache, dry mouth, sense of unreality, feeling high, dizzy, sleepy, and sad occurred more often. However, anxiety levels were lower after dronabinol intake, and confusion was not connected with treatment [54].

Another study evaluating the hypothesis of dronabinol potential in anorexia management is estimated to be completed in the 2021 open-label clinical trial. In this study patients diagnosed with head, neck, lung, or pancreatic cancer will undergo pharmacotherapy in order to decrease the suffering and distress associated with eating during cancer treatment and improve the quality of life of patients. The dronabinol pharmacotherapy will be compared to Dexamethasone, Mirtazapine, and behavioral therapy [55]. 


\section{Dronabinol dementia neuropsychiatric symptoms treatment}

Dronabinol may also be effective in managing dementia-related neuropsychiatric symptoms. Balance and gait were assessed in a double-blind, crossover RCT. Results suggest that $3 \mathrm{mg}$ Namisol intake tended to be associated with balance and gait disturbance. It increased body sway when patients were standing with eyes closed. Pitch angular displacement during preferred speed walking, stride length during walking at preferred speed, and gain velocity increased significantly after THC administration. No AEs were connected to the treatment [56].

\section{The abuse potential of dronabinol}

Published in 2018 a double-blind, crossover RCT abuse potential of single oral doses of Epidiolex (750 mg, $1500 \mathrm{mg}$, or $4500 \mathrm{mg}$ ), alprazolam (2 mg), and dronabinol (10 mg or $30 \mathrm{mg}$ ) was evaluated among 43 healthy recreational polydrug users. In the study the largest increase in mean Drug-Liking VAS, Drug-Liking VAS Emax and Take Drug Again VAS scores were observed for dronabinol. Moreover, synthetic cannabinoid dosages were associated with a decrease in mean Alertness/Drowsiness VAS scores. Cognitive and psychomotor effects for $10 \mathrm{mg}$ doses of dronabinol did not differ from placebo, whereas $30 \mathrm{mg}$ pharmacotherapy caused a slight decrease in their performance. AEs were the highest for dronabinol, although their intensity was moderate or mild (most often somnolence, euphoric mood, nausea). The authors indicated a correlation between synthetic cannabinoid therapy and increased HR with no relevant changes in hematology, biochemistry, or urinalysis parameters [57].

\section{Dronabinol impact on hemodynamics}

The effectiveness of a single dose of $5 \mathrm{mg}$ dronabinol was also evaluated on change in optic nerve head blood flow among 24 healthy adults. The conclusions of this study may be promising for patients with impairment of ocular hemodynamics. Although dronabinol had no effect on Intraocular Pressure (IOP), MAP, and Ocular Perfusion Pressure (OPP), it induced an increase in ocular blood flow. The study was conducted on healthy subjects, so the author indicated that it remains unknown whether dronabinol would counteract the vasoconstrictor response of the optic nerve head vasculature to an increase in OPP among people with vascular ocular disease or glaucoma. An additional limitation mentioned by the authors is the usage of a single dose of dronabinol so long-term administration effects cannot be concluded. There were not observed any adverse and psychoactive effects [58].

\section{Other therapeutic use of dronabinol}

A parallel, quadruple-blind RCT, which has an estimated completion date for 2022 will focus on an effect of alternative $5 \mathrm{mg}-10 \mathrm{mg}$ daily dose of Marinol for agitation treatment in 160 patients with Alzheimer's Disease (AD). CB1R agonism of oral cannabinoids may decrease combativeness, yelling, pacing, lack of cooperation with care, insomnia, and restlessness, while CB2R agonism can reduce central and peripheral inflammation. Possible adverse effects will be recorded [59].

A cross-over, double-masked RCT will try to prove the hypothesis that $10-20 \mathrm{mg}$ of dronabinol can be a useful treatment option for adults diagnosed with Sickle Cell Disease (SCD). The study will be conducted on 30 patients and is scheduled to end in 2021. Reduction in pain interference, scores, and quality, decrease in markers of inflammation and opioid intake, improvement in stiffness, the intensity of nausea and vomiting, sleep, mood, anxiety, and social functioning will be observed [60].

Another promising study, which is estimated to end in 2022 is going to evaluate the hypothesis that oral dronabinol improves nightmares and other PTSD symptoms to a greater extent than placebo. This 10-week, double-blind, parallel RCT will check the frequency and intensity of nightmares, measured with the Clinician-Administered PTSD Scale-IV (CAPS-IV) [61].

\section{Adverse events, side effects, and toxicity}

Among 29 included studies, in 15 there were not observed any AEs. The lack of side effects is considered to be caused by short therapy duration. In the remaining 14 articles, there were registered only two studies, which reported a few SARs. The most common AEs include dizziness, somnolence, heart arrhythmia, dry mouth, euphoric mood, and nausea. The side effects were related to long therapy duration and high cannabinoid dose administration. In conclusion, according to analyzed studies, dronabinol pharmacotherapy is characterized by good safety and tolerability.

\section{Conclusions}

This article reviewed plentiful studies which highlighted the promising potential of dronabinol 
as a therapeutic agent. According to reviewed studies, synthetic cannabinoids can be considered as a therapeutic option for cannabis use disorder, OSA, TS, relieving stress, and anorexia treatment. However, it did not present potential in opioid addiction, nausea and vomiting, and dementia neuropsychiatric symptoms management. Results of clinical trials, which focused on the analgesic potential of dronabinol are inconsistent. Additionally, dronabinol's impact on brain function was reviewed specifically on cognitive reappraisal, memory retrieval, extinction recall, the intoxication of PFC, and the threat extinction network. Undoubtedly, a beneficial characteristic of dronabinol is its relatively low toxicity. The side effects reported in the studies, if appeared, were mostly mild to moderate. The most common were dizziness, somnolence, heart arrhythmia, dry mouth, euphoric mood, and nausea. Ongoing clinical trials should give a wider view of the thesis about the therapeutic potential of dronabinol and about the long-term effects of therapy.

\section{Literature}

1. National Center for Biotechnology Information (2020). PubChem Compound Summary for CID 16078, Dronabinol. Retrieved October 4, 2020 from https://pubchem.ncbi.nlm.nih.gov/compound/ Dronabinol. (access 15.05.2021).

2. Sheikh NK, Dua A. Cannabinoids. 2020 Jun 22. In: StatPearls [Internet]. Treasure Island (FL): StatPearls Publishing; 2020 Jan-. PMID: 32310522

3. Basavarajappa BS, Shivakumar M, Joshi V, Subbanna S. Endocannabinoid system in neurodegenerative disorders. $J$ Neurochem. 2017; 142(5): 624-648. doi: 10.1111/jnc.14098. Epub 2017 Jul 5. PMID: 28608560; PMCID: PMC5669051.

4. Svízenská I, Dubový P, Sulcová A. Cannabinoid receptors 1 and 2 (CB1 and CB2), their distribution, ligands and functional involvement in nervous system structures--a short review. Pharmacol Biochem Behav. 2008; 90(4): 501-511. doi: 10.1016/j. pbb.2008.05.010. PMID: 18584858.

5. Di Marzo V, Bifulco M, De Petrocellis L. The endocannabinoid system and its therapeutic exploitation. Nat Rev Drug Discov. 2004 Sep; 3(9): 771-784. doi: 10.1038/nrd1495. PMID: 15340387.

6. Grotenhermen F. Pharmacokinetics and pharmacodynamics of cannabinoids. Clin Pharmacokinet. 2003; 42(4): 327-360. doi: 10.2165/00003088-200342040-00003. PMID: 12648025.

7. Oh DA, Parikh N, Khurana V, Cognata Smith C, Vetticaden S. Effect of food on the pharmacokinetics of dronabinol oral solution versus dronabinol capsules in healthy volunteers. Clin Pharmacol. 2017 Jan; 11; : 9: 9-17. doi: 10.2147/CPAA.S119676. PMID: 28138268 ; PMCID: PMC5238805.

8. Drugs.com; Dronabinol; https://www.drugs.com/ingredient/dronabinol.html (access 15.05.2021).

9. Cannnabis Medicinales, International for Cannabinoid Medicines https://www.cannabis-med.org/index.php?tpl=page\&id=236\&ln$\mathrm{g}=\mathrm{it}$ (access 15.05.2021)

10. Drug Free Australia Brief to Australian Parliamentarians Medical Cannabis; https://www.dalgarnoinstitute.org.au/images/resources/pdf/aod/cannabis-documents/Drug-Free-Australia-Briefto-Australian-Parliamentarians-Medical-Cannabis.pdf (access 15.05.2021).

11. LiverTox: Clinical and Research Information on Drug-Induced Liver Injury [Internet]. Bethesda (MD): National Institute of Diabetes and Digestive and Kidney Diseases; 2012. Dronabinol. [Updated 2018 Jan 15].

12. Murad MH, Mustafa RA, Schünemann HJ, Sultan S, Santesso N. Rating the certainty in evidence in the absence of a single estimate of effect. Evid Based Med. 2017 Jun; 22(3): 85-87. doi: 10.1136/ ebmed-2017-110668. Epub 2017 Mar 20. PMID: 28320705; PMCID: PMC5502230.
13. Levin FR, Mariani JJ, Pavlicova M, Brooks D, Glass A, Mahony A, Nunes EV, Bisaga A, Dakwar E, Carpenter KM, Sullivan MA, Choi JC. Dronabinol and lofexidine for cannabis use disorder: A randomized, double-blind, placebo-controlled trial. Drug Alcohol Depend. 2016 Feb 1; 159(2): 53-60. doi: 10.1016/j.drugalcdep.2015.11.025. Epub 2015 Nov 27. PMID: 26711160; PMCID: PMC4729291.

14. https://clinicaltrials.gov/ct2/show/NCT01394185 (access 15.05.2021).

15. https://clinicaltrials.gov/ct2/show/NCT00893074 (access 15.05.2021).

16. Vandrey R, Stitzer ML, Mintzer MZ, Huestis MA, Murray JA, Lee D. The dose effects of short-term dronabinol (oral THC) maintenance in daily cannabis users. Drug Alcohol Depend. 2013 Feb 1; 128(12): 64-70. doi: 10.1016/j.drugalcdep.2012.08.001. Epub 2012 Aug 22. PMID: 22921474; PMCID: PMC3546149.

17. https://clinicaltrials.gov/ct2/show/NCT01598896 (access 15.05.2021).

18. https://clinicaltrials.gov/ct2/show/NCT00480441 (access 15.05.2021).

19. Haney M, Hart CL, Vosburg SK, Nasser J, Bennett A, Zubaran C, Foltin RW. Marijuana withdrawal in humans: effects of oral THC or divalproex. Neuropsychopharmacology 2004; 29(1):158-170. doi: 10.1038/sj.npp.1300310. PMID: 14560320.

20. Schlienz NJ, Lee DC, Stitzer ML, Vandrey R. The effect of high-dose dronabinol (oral THC) maintenance on cannabis self-administration. Drug Alcohol Depend. 2018 Jun 1; 187(6): 254-260. doi: 10.1016/j.drugalcdep.2018.02.022. Epub 2018 Apr 17. PMID: 29689485; PMCID: PMC5959782.

21. https://clinicaltrials.gov/ct2/show/NCT03560934 (access 15.05.2021).

22. https://clinicaltrials.gov/ct2/show/NCT01964404 (access 15.05.2021).

23. Lofwall MR, Babalonis S, Nuzzo PA, Elayi SC, Walsh SL. Opioid withdrawal suppression efficacy of oral dronabinol in opioid dependent humans. Drug Alcohol Depend. 2016 Jul 1; 164(7): 143-150. doi: 10.1016/j.drugalcdep.2016.05.002. Epub 2016 May 10. PMID: 27234658; PMCID: PMC4910823.

24. https://clinicaltrials.gov/ct2/show/NCT03766269 (access 15.05.2021).

25. https://clinicaltrials.gov/ct2/show/NCT04298528 (access 15.05.2021).

26. https://clinicaltrials.gov/ct2/show/NCT00743119 (access 15.05.2021).

27. Cooper ZD, Comer SD, Haney M. Comparison of the analgesic effects of dronabinol and smoked marijuana in daily marijuana smokers. Neuropsychopharmacology 2013 Sep; 38(10): 1984-1992. doi: 10.1038/npp.2013.97. Epub 2013 Apr 22. PMID: 23609132; PMCID: PMC3746706.

28. Malik Z, Bayman L, Valestin J, Rizvi-Toner A, Hashmi S, Schey $R$. Dronabinol increases pain threshold in patients with functional chest pain: a pilot double-blind placebo-controlled trial. Dis Esophagus. 2017; 30(2): 1-8. doi: 10.1111/dote.12455. PMID: 26822791.

29. https://clinicaltrials.gov/ct2/show/NCT01598207 (access 15.05.2021).

30. Reichenbach ZW, Sloan J, Rizvi-Toner A, Bayman L, Valestin J, Schey R. A 4-week pilot study with the cannabinoid receptor agonist dronabinol and its effect on metabolic parameters in a randomized trial. Clin Ther. 2015; 37(10): 2267-2274. doi: 10.1016/j. clinthera.2015.07.023. Epub 2015 Aug 14. PMID: 26283236.

31. Schimrigk S, Marziniak M, Neubauer C, Kugler EM, Werner G, Abramov-Sommariva D. Dronabinol Is a Safe Long-Term Treatment Option for Neuropathic Pain Patients. Eur Neurol. 2017; 78(5-6): 320-329. doi: 10.1159/000481089. Epub 2017 Oct 26. PMID: 29073592; PMCID: PMC5804828.

32. de Vries M, Van Rijckevorsel DC, Vissers KC, Wilder-Smith $\mathrm{OH}$, Van Goor H. Single dose delta-9-tetrahydrocannabinol in chronic pancreatitis patients: analgesic efficacy, pharmacokinetics and tolerability. Br J Clin Pharmacol. 2016; 81(3): 525-537. doi: 10.1111/bcp.12811. Epub 2016 Jan 17. PMID: 26505163; PMCID: PMC4767190

33. de Vries M, van Rijckevorsel DCM, Vissers KCP, Wilder-Smith OHG, van Goor H; Pain and Nociception Neuroscience Research Group. Tetrahydrocannabinol Does Not Reduce Pain in Patients With Chronic Abdominal Pain in a Phase 2 Placebo-controlled Study. Clin Gastroenterol Hepatol. 2017; 15(7): 1079-1086.e4. doi: 10.1016/j.cgh.2016.09.147. Epub 2016 Oct 5. PMID: 27720917.

34. van Amerongen G, Siebenga P, de Kam ML, Hay JL, Groeneveld GJ. Effect profile of paracetamol, $\Delta 9$-THC and promethazine using an evoked pain test battery in healthy subjects. Eur J Pain. 2018 Aug; 
22(7): 1331-1342. doi: 10.1002/ejp.1222. Epub 2018 Apr 25. PMID 29635857.

35. Babalonis S, Lofwall MR, Sloan PA, Nuzzo PA, Fanucchi LC, Walsh SL. Cannabinoid modulation of opioid analgesia and subjective drug effects in healthy humans. Psychopharmacology (Berl) 2019 236(11): 3341-3352. doi: 10.1007/s00213-019-05293-1. Epub 2019 Jun 15. PMID: 31201479; PMCID: PMC6832798.

36. https://clinicaltrials.gov/ct2/show/NCT03604341 (access 15.05.2021)

37. Colwill AC, Alton K, Bednarek PH, Bayer LL, Jensen JT, Garg B, Beardsworth K, Edelman A. Cannabinoids for Pain Control During Medical Abortion: A Randomized Controlled Trial. Obstet Gynecol. 2020; 135(6): 1289-1295. doi: 10.1097/AOG.0000000000003850. PMID: 32459420

38. https://clinicaltrials.gov/ct2/show/NCT04346407 (access 15.05.2021).

39. https://clinicaltrials.gov/ct2/show/NCT04099355 (access 15.05.2021).

40. Gorka SM, Phan KL, Lyons M, Mori S, Angstadt M, Rabinak CA. Cannabinoid Modulation of Frontolimbic Activation and Connectivity During Volitional Regulation of Negative Affect. Neurop sychopharmacology 2016 Jun; 41(7): 1888-1896. doi: 10.1038 npp.2015.359. Epub 2015 Dec 9. PMID: 26647971; PMCID: PMC4869058.

41. Doss MK, Weafer J, Gallo DA, de Wit H. $\Delta 9$-Tetrahydrocannabino at Retrieval Drives False Recollection of Neutral and Emotional Memories. Biol Psychiatry. 2018 Nov 15; 84(10): 743-750. doi: 10.1016/j.biopsych.2018.04.020. Epub 2018 May 9. PMID 29884456.

42. Rabinak CA, Peters C, Marusak HA, Ghosh S, Phan KL. Effects of acute $\Delta 9$-tetrahydrocannabinol on next-day extinction recall is mediated by post-extinction resting-state brain dynamics. Neuropharmacology 2018; 143(12): 289-298. doi: 10.1016/j.neuropharm.2018.10.002. Epub 2018 Oct 3. PMID: 30291940; PMCID: PMC6269194.

43. https://clinicaltrials.gov/ct2/show/NCT03008005 (access 15.05.2021)

44. Gilman JM, Yücel MA, Pachas GN, Potter K, Levar N, Broos H, Manghis EM, Schuster RM, Evins AE. Delta-9-tetrahydrocannabino intoxication is associated with increased prefrontal activation as assessed with functional near-infrared spectroscopy: A report of a potential biomarker of intoxication. Neuroimage 2019; 197(8) 575-585. doi: 10.1016/j.neuroimage.2019.05.012. Epub 2019 May 7. PMID: 31075393; PMCID: PMC6590705.

45. https://clinicaltrials.gov/ct2/show/NCT03655717 (access 15.05.2021)

46. Hammoud MZ, Peters C, Hatfield JRB, Gorka SM, Phan KL, Milad $\mathrm{MR}$, Rabinak CA. Influence of $\Delta 9$-tetrahydrocannabinol on long-term neural correlates of threat extinction memory retention in humans. Neuropsychopharmacology 2019 Sep; 44(10): 17691777. doi: 10.1038/s41386-019-0416-6. Epub 2019 May 16. PMID 31096264; PMCID: PMC6784991.
47. https://clinicaltrials.gov/ct2/show/NCT04231643 (access 15.05.2021)

48. https://clinicaltrials.gov/ct2/show/NCT01755091 (access 15.05.2021).

49. Carley DW, Prasad B, Reid KJ, Malkani R, Attarian H, Abbott SM, Vern B, Xie H, Yuan C, Zee PC. Pharmacotherapy of Apnea by Cannabimimetic Enhancement, the PACE Clinical Trial: Effects of Dronabinol in Obstructive Sleep Apnea. Sleep. 2018; 41(1):zsx184. doi: 10.1093/sleep/zsx184. PMID: 29121334; PMCID: PMC5806568.

50. https://clinicaltrials.gov/ct2/show/NCT00757822 (access 15.05.2021).

51. https://clinicaltrials.gov/ct2/show/NCT03984214 (access 15.05.2021).

52. https://clinicaltrials.gov/ct2/show/NCT03066193 (access 15.05.2021).

53. Childs E, Lutz JA, de Wit H. Dose-related effects of delta-9-THC on emotional responses to acute psychosocial stress. Drug Alcohol Depend. 2017 Aug 1;177:136-144. doi: 10.1016/j.drugalcdep.2017.03.030. Epub 2017 May 30. PMID: 28599212; PMCID: PMC6349031.

54. Weltens N, Depoortere I, Tack J, Van Oudenhove L. Effect of acute $\Delta 9$-tetrahydrocannabinol administration on subjective and metabolic hormone responses to food stimuli and food intake in healthy humans: a randomized, placebo-controlled study. Am J Clin Nutr. 2019; 109(4): 1051-1063. doi: 10.1093/ajen/nqz007. PMID: 30949710.

55. https://clinicaltrials.gov/ct2/show/NCT04155008 (access access 15.05.2021).

56. van den Elsen GA, Tobben L, Ahmed AI, Verkes RJ, Kramers C, Marijnissen RM, Olde Rikkert MG, van der Marck MA. Effects of tetrahydrocannabinol on balance and gait in patients with dementia: A randomised controlled crossover trial. J Psychopharmacol. 2017; 31(2): 184-191. doi: 10.1177/0269881116665357. Epub 2016 Sep 27. PMID: 27624148.

57. Schoedel KA, Szeto I, Setnik B, Sellers EM, Levy-Cooperman N, Mills C, Etges T, Sommerville K. Abuse potential assessment of cannabidiol (CBD) in recreational polydrug users: A randomized, double-blind, controlled trial. Epilepsy Behav. 2018; 88(11): 162171. doi: 10.1016/j.yebeh.2018.07.027. Epub 2018 Oct 2. PMID 30286443 .

58. Hommer N, Kallab M, Szegedi S, Puchner S, Stjepanek K, Bauer M, Werkmeister RM, Schmetterer L, Abensperg-Traun M, Garhöfer G, Schmidl D. The Effect of Orally Administered Dronabinol on Optic Nerve Head Blood Flow in Healthy Subjects-A Randomized Clinical Trial. Clin Pharmacol Ther. 2020 Jul; 108(1): 155-161. doi: 10.1002/cpt.1797. Epub 2020 Feb 23. PMID: 31977076; PMCID: PMC7325313.

59. https://clinicaltrials.gov/ct2/show/NCT02792257 (access 15.05.2021)

60. https://clinicaltrials.gov/ct2/show/NCT03978156 (access 15.05.2021)

61. https://clinicaltrials.gov/ct2/show/NCT04448808 (access 15.05.2021). 